Original Article

\title{
Reliability and reproducibility of interapical distance assessment of the lateral deviation of vertebrae in scoliosis
}

\author{
Jeong Hoon Lim, MD, PhD ${ }^{1)}$, Jongmin Lee, MD, PhD ${ }^{2)}$, Seong-Eun Koh, MD, $\mathrm{PhD}^{2)}$, \\ IN-SiK LEE, MD, $\mathrm{PhD}^{2}$ * $^{*}$ \\ 1) Department of Medicine, National University Hospital, Yong Loo Lin School of Medicine, National \\ University of Singapore, Singapore \\ 2) Department of Rehabilitation Medicine, Konkuk University Medical Center, Konkuk University \\ School of Medicine: 120-1 Neungdongro, Gwangjingu, Seoul 143-729, Republic of Korea
}

\begin{abstract}
Purpose] The purpose of this study was to investigate the interobserver reliability and intraobserver reproducibility of interapical distance (IAD) and to analyze its correlation with the Cobb angle (CA). [Subjects and Methods] IAD, a handy tool for assessment of the lateral deviation of vertebrae with a metric scale, was defined as the horizontal distance between one apical vertebra and its counterpart, the opposite apical vertebra in the case of a double curve and the farthest vertebra in the case of a single curve. Fifty full-length, standing anteroposterior radiographs of "idiopathic scoliosis" were reviewed. Three investigators independently measure the CA and IAD at the same time and remeasured the IAD on the same radiograph a week later. [Results] There was no interobserver difference (reliability) in the measurement of IAD or statistical differences in intraobserver reproducibility for each observer. IAD was well correlated with the $\mathrm{CA}$ for each observer $(\mathrm{r}=0.765, \mathrm{r}=0.737$, and $\mathrm{r}=0.764)$. [Conclusion] IAD is useful when assessing lateral deviation in scoliosis and may be a reliable and reproducible index that is well correlated with the CA, and it can be used as a supplementary measure to describe the overall derangement of scoliosis in the coronal plane.

Key words: Scoliosis, Interapical distance, Lateral deviation
\end{abstract}

(This article was submitted Nov. 4, 2014, and was accepted Dec. 11, 2014)

\section{INTRODUCTION}

Scoliosis is a lateral curvature of the spine that is the result of various pathologic mechanisms including congenital vertebral defect, back pain, neurologic disorder, posture imbalance, and idiopathic causes. To explain idiopathic scoliosis, several hypotheses, such as disproportion of vertebraneural growth ${ }^{1,2)}$ or interference of muscles and ligaments with rapidly growing vertebrae in the second growth spurt ${ }^{3}$, have been suggested. Body morphology has been reported to affect the center of pressure position in idiopathic scoliosis ${ }^{4}$.

From a clinical perspective, the correlation between radiographic parameters and the patient's physical as well as functional status has been used in the decision-making process for selection of treatment options. To acquire radiographic parameters, the scoliotic curve should be evaluated in the coronal, sagittal, and axial planes because it is a threedimensional deformity. Hitherto, diverse measuring tools

*Corresponding Author. In-Sik Lee (E-mail: mdlis@kuh. ac.kr)

C2015 The Society of Physical Therapy Science. Published by IPEC Inc. This is an open-access article distributed under the terms of the Creative Commons Attribution Non-Commercial No Derivatives (by-ncnd) License $<$ http://creativecommons.org/licenses/by-nc-nd/3.0/>. covering each plane have been presented. For the coronal plane, the representative view of scoliosis, the Cobb angle (CA) has been the primary radiographic measure used for evaluation and treatment of scoliosis ${ }^{5}$. The reproducibility of the CA has been well defined with acceptable variations between measurements and excellent overall reliability as well ${ }^{6-8)}$

In daily practice in clinics and gyms, a quick and easy technique is needed to evaluate the lateral deviation of vertebrae and spinal balance as a whole. So far, noninvasive methods such as measurement with a scoliometer and topography have been used by practitioners because they are easy to apply $\left.{ }^{9}, 10\right)$. However, these techniques cannot be used interchangeably and are not appropriate for measurement of lateral deviation in scoliosis ${ }^{11)}$.

Despite the aforementioned merits in scoliosis assessment, the CA which is based on angular measurement of curves, has some limitation in evaluating lateral deviation of vertebrae with a metric scale. Given that severity of scoliosis could be presented with not only the angle but also the width of the scoliotic spine, a reliable metric index representing lateral deviation of the spine may provide a new perspective and information that complements the CA.

Therefore, we are suggesting a new concept, interapical distance (IAD), a simple and accurate index that can represent the general picture of lateral deviation before and 


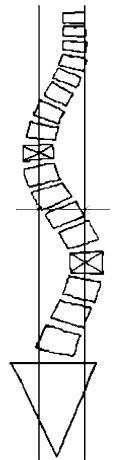

A

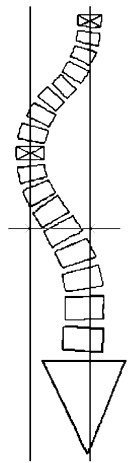

B
Fig. 1. Measurement of interapical distance for a double curve spine (A) and for a single curve spine (B)

after treatment of scoliosis and can be readily applicable to all types of scoliosis, irrespective of curve shape or compensation. IAD is defined as the horizontal distance in millimeters from the midpoint of the apical body or disc to its counterpart, the opposite apical body or disc, in a double (or triple) curve (Fig. 1A) and the farthest vertebral body or disc between $\mathrm{T} 1$ and L5 in a single curve (Fig. 1B).

The purpose of this study was to evaluate the interobserver reliability and intraobserver reproducibility of the IAD and check its correlation with the CA, the most commonly used radiographic parameter for assessment of the severity of scoliotic deformity.

\section{SUBJECTS AND METHODS}

The Institutional Review Board of Konkuk University Medical Center approved this retrospective radiographic study, and there was no adverse effect on subjects. In this study, the medical records and radiographic studies of 204 patients that had been diagnosed with "scoliosis" at Konkuk University Medical Center spine clinic from 1 June 2007 to 31 May 2011 were retrieved initially, but only patients ranging from 10 to 17 years of age were included. After reviewing the medical records, patients with the following were excluded: vertebral defect, back pain, or neuromuscular disorder.

Patients' full-length, standing anteroposterior radiographs from their the first visit to the University Medical Center were examined by 3 independent observers, who had more than ten years of experience in scoliosis treatment, using the PACS software, Centricity Enterprise Web V3.0 (GE Healthcare, Fairfield, CT, USA). In terms of the angular threshold for definition of scoliosis, we referred to the 2011 SOSORT guidelines ${ }^{12)}$, which declare that the diagnosis of scoliosis should not be made under $10^{\circ}$ of scoliosis and that the recognized measurement error in measuring Cobb angles is $5^{\circ}$. In this study, It was determined that the apex vertebra should be located below $\mathrm{T} 1$ and that the CA of the curve measured by the observer should be equal to or above $15^{\circ}$ unanimously. Three observers independently measured the CA according to the SRS definition ${ }^{13)}$ and measured the IAD at the same time. Among the 204 patients, 50 patients with
Table 1. Demographic characteristics and scoliosis classification of subjects

\begin{tabular}{lc}
\hline Gender & Male (8) \\
& Female (42) \\
Age (yrs) & $15.2 \pm 1.6$ \\
King type* & King 1 (22) \\
& King 2 (11) \\
King 3 (11) \\
King 4 (5) \\
King 5 (1) \\
\hline *Only those curves with a Cobb angle \\
of more than 15 degrees were counted as \\
major or minor curves.
\end{tabular}

"adolescent idiopathic scoliosis" were ultimately included, and their demographic and clinical characteristics are shown in Table 1. Three observers independently measured the CA according to the SRS definition ${ }^{13)}$ and the IAD at the same time. After one week, the IAD was measured again on the same radiograph. All measurement results from the 3 observers were included, and there were no missing data.

To select the appropriate analytic method for identification of the interaction between rater and rating time point of IAD measurement, Mauchly's sphericity test was performed. Regarding the sphericity test results, interobserver and intraobserver interactions with the rating time points were analyzed using MANOVA and ANOVA respectively.

To evaluate the interobserver reliability and intraobserver reproducibility of IAD measurement, ANOVA and the paired $t$ test were used accordingly. The correlation between the CA of the major curve and the IAD was analyzed. Data were processed using the SPSS v17.0 (SPSS Inc., Chicago, IL, USA), and a p-value less than 0.05 was considered statistically significant.

\section{RESULTS}

There was no significant interobserver or intraobserver interaction with the rating time points of a one-week interval (MANOVA, $\mathrm{p}=0.349, \mathrm{p}=0.347$ ). IAD was measured two times, one week apart, by 3 observers, and the values are presented in Table 2. The IAD values for the first measurement (IAD1) were $28.5 \mathrm{~mm}, 27.9 \mathrm{~mm}$, and $28.4 \mathrm{~mm}$ for observer 1 , observer 2 , and observer 3 . The IAD values for the second measurement (IAD2) were $28.0 \mathrm{~mm}, 27.8 \mathrm{~mm}$, and $28.2 \mathrm{~mm}$, respectively. To assess the intraobserver reproducibility, the paired t test was performed, and there was no statistical difference in two trials of IAD measurement by the same observer (observer $1, \mathrm{p}=0.510$; observer $2, \mathrm{p}=0.908$; observer $3, \mathrm{p}=0.614$ ), which implied stability in the application of this method regardless repeated trials. To evaluate the interobserver difference in the measurement of the IAD, the mean IAD was calculated for each observer with the following formula: (IAD1+IAD2)/2; the mean values were $28.3 \mathrm{~mm}$ (observer 1), $28.0 \mathrm{~mm}$ (observer 2), and $28.1 \mathrm{~mm}$ (observer 3). For these data, ANOVA was performed and showed no difference $(\mathrm{p}=0.981)$ between observers, which supported the interobserver reliability. For each observer, the $\mathrm{CA}$ and mean IAD, the average value of two trials of IAD 
Table 2. Measurement of interapical distance and Cobb angle by three observers

\begin{tabular}{|c|c|c|c|c|}
\hline \multirow[t]{2}{*}{ Parameter } & \multicolumn{3}{|c|}{ Observer } & \multirow{2}{*}{$\begin{array}{c}\text { Interobserver difference } \\
\text { ANOVA, p-value }\end{array}$} \\
\hline & 1 & 2 & 3 & \\
\hline IAD1 (mm) & 28.5 & 27.9 & 28.4 & \\
\hline $95 \% \mathrm{CI}$ & $24.5-32.5$ & $24.2-31.5$ & $24.8-32.0$ & \\
\hline IAD2 (mm) & 28.0 & 27.8 & 28.2 & \\
\hline $95 \% \mathrm{CI}$ & $24.3-31.8$ & $24.5-31.1$ & $24.4-31.9$ & \\
\hline Paired t-test, $\mathrm{p}$-value & 0.510 & 0.908 & 0.614 & \\
\hline Mean IAD (mm) & 28.3 & 28.0 & 28.1 & 0.981 \\
\hline $95 \% \mathrm{CI}$ & $24.5-31.1$ & $24.6-31.4$ & $24.4-31.9$ & \\
\hline CA (degree) & 27.5 & 27.2 & 27.0 & \\
\hline $95 \% \mathrm{CI}$ & $24.8-30.3$ & $24.6-29.7$ & $24.4-29.6$ & \\
\hline Correlation coefficient & 0.765 & 0.737 & 0.764 & \\
\hline p-value & $\leq 0.001^{*}$ & $\leq 0.001^{*}$ & $\leq 0.001 *$ & \\
\hline
\end{tabular}

IAD: interapical distance; IAD1: first measurement of IAD; IAD2: second measurement of IAD; mean IAD, (IAD1+IAD2)/2; CA, Cobb angle.

*Statistically significant

measurement, were well correlated (observer $1, r=0.765$, $\mathrm{p} \leq 0.001$; observer $2, \mathrm{r}=0.737, \mathrm{p} \leq 0.001$; observer $3, \mathrm{r}=0.764$, $\mathrm{p} \leq 0.001)$.

\section{DISCUSSION}

It is evident that lateral deviation of the vertebra in scoliosis is the result of multidimensional forces that include lateral bending force, axial rotation, and kyphotic or lordotic array in the sagittal plane. To elucidate the interaction of these variables, many indices and sophisticated protocols using plane radiographs or even computed tomography have been introduced. In a coronal plane, the $\mathrm{CA}$, apical vertebral lateral deviation (AVLD), and coronal balance (CB) have been used frequently in the evaluation of scoliosis.

Though IAD evaluates lateral deviation with a metric scale, it is highly correlated with the CA, the established indicator of scoliosis severity in terms of angular measurement, as shown in Table 2. This finding implies that the IAD has clinical applicability and that it can complement the CA. Meanwhile, the IAD is not dependent on the spinal compensation in scoliosis because there is no significant statistical correlation between the IAD and $\mathrm{CB}$, as shown by another investigator's analysis (LJH: data not shown). Regardless of curve compensation, easy applicability to diverse types of scoliosis would be the strength of the IAD.

AVLD, which was initially introduced as a means of distinguishing true King type 2 curves from similar ones ${ }^{14)}$, is applicable to quantification of apical displacement and is known to have a good interobserver reliability ${ }^{8}$. Besides, AVLD and its ratio are useful when making surgical decisions about fusion level, etc ${ }^{15)}$. AVLD is defined as the distance from the central sacral line (CSL) to the midpoint of the apical body. In a decompensated patient, it should be measured from the CSL for the thoracolumbar and lumbar spine, and from the $\mathrm{C} 7$ plumb line (C7PL) for the thoracic spine $^{13)}$

However, considering the aforementioned stipulation regarding the curve type and shape, AVLD seems to be

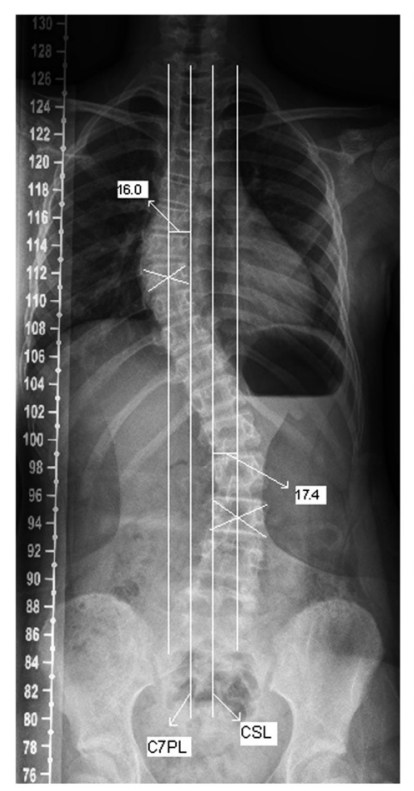

A

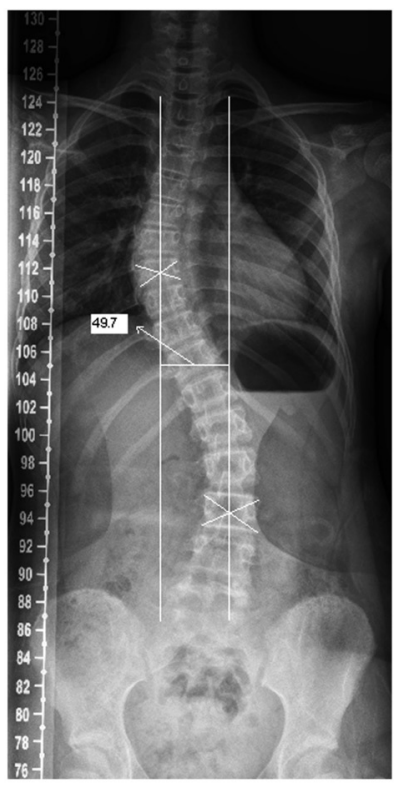

B
Fig. 2. Measurement of the lateral deviation of the scoliotic spine: apical vertebral lateral deviation method (A) and interapical distance method (B).

C7PL: C7 plumb line; CSL: central sacral line

complicated in some cases. For example, when it comes to a decompensated double curve with two apices in the thoracic and lumbar regions as in Lenke type 3 and 6 or King type 1 and 2, AVLD should be measured twice from different base lines, the CSL or C7PL (Fig. 2A). In the case of Lenke type 4 (triple major curve), it should be measured three times from different base lines for each corresponding apex, such as C7PL-apex, CSL-apex, and CSL-apex again. On top of that, AVLD does not correlate well with the CA given that spinal curves with the same CA can have significantly different AVLD values ${ }^{16)}$. 
Should the scoliotic curve form a sine wave, AVLD would correspond to the peak-to-base line amplitude. In decompensated scoliosis, the base line should be defined differently depending on the curve shape and corresponding apex: CSL for the thoracolumbar and lumbar spine and C7PL for the thoracic spine as per SRS definition ${ }^{13)}$. On the other hand, IAD could exhibit a peak-to-peak amplitude irrespective of the variability of the curve shape, so there is no need to consider the base line. In some way, IAD and AVLD can supplement each other. In the same case as shown above for AVLD measurement, IAD is illustrated according to its definition in Fig. 2B.

This study has several limitations. Firstly, it used only 3 observers who had more than ten years of experience in scoliosis treatment. Usually, radiographic studies include observers at different training levels to exclude the effect of experience. Therefore, we cannot address the reliability of this method with less experienced observers. However, the measuring techniques and radiographic landmarks used in this study are simple, so less experienced hands should be able to learn this protocol easily. Secondly, a limited number of patients with idiopathic scoliosis were included in this study. However, IAD is expected to be workable in various kinds of scoliosis in principle such as degenerative scoliosis, vertebral defect, neurological disorder, and so forth. It can also be used as a measuring tool to observe the clinical course of scoliosis and post-treatment follow-up. In this context, it is worthwhile to utilize the IAD for other forms of scoliosis with different etiologies in the future. Lastly, this study suggests the promise of IAD as a measuring tool for lateral deviation of the scoliotic spine, but does not show the actual correlation with patients' symptoms. This limitation does not only apply to IAD given that there has been much controversy over the correlation between other radiographic parameters including the CA and patient health status ${ }^{17-19)}$. Despite these limitations, the results of this study seem to support the applicability and significance of IAD for measurement of the lateral deviation of vertebrae in scoliosis.

In conclusion, IAD was found to be a reliable and reproducible tool for the assessment of the lateral deviation of the spine in an anteroposterior radiograph and was well correlated with the CA. IAD seems to be a simple and useful measure for evaluation of lateral derangement in scoliosis.

\section{ACKNOWLEDGEMENT}

This work was supported by Konkuk University.

\section{REFERENCES}

1) Roth M: Idiopathic scoliosis from the point of view of the neuroradiologist. Neuroradiology, 1981, 21: 133-138. [Medline]

2) Roth M: Idiopathic scoliosis caused by a short spinal cord. Acta Radiol Diagn (Stockh), 1968, 7: 257-271. [Medline]

3) Yang Z, Xie Y, Li M: Three-dimensional spring model: a new hypothesis of pathogenesis of adolescent idiopathic scoliosis. Med Hypotheses, 2009, 73: 709-713. [Medline] [CrossRef]

4) Allard P, Chavet P, Barbier F, et al.: Effect of body morphology on standing balance in adolescent idiopathic scoliosis. Am J Phys Med Rehabil, 2004, 83: 689-697. [Medline] [CrossRef]

5) Lenke LG, Betz RR, Harms J, et al.: Adolescent idiopathic scoliosis: a new classification to determine extent of spinal arthrodesis. J Bone Joint Surg Am, 2001, 83-A: 1169-1181. [Medline]

6) Carman DL, Browne RH, Birch JG: Measurement of scoliosis and kyphosis radiographs. Intraobserver and interobserver variation. J Bone Joint Surg Am, 1990, 72: 328-333. [Medline]

7) Morrissy RT, Goldsmith GS, Hall EC, et al.: Measurement of the Cobb angle on radiographs of patients who have scoliosis. Evaluation of intrinsic error. J Bone Joint Surg Am, 1990, 72: 320-327. [Medline]

8) Kuklo TR, Potter BK, Polly DW Jr, et al.: Reliability analysis for manual adolescent idiopathic scoliosis measurements. Spine, 2005, 30: 444-454. [Medline] [CrossRef]

9) Amendt LE, Ause-Ellias KL, Eybers JL, et al.: Validity and reliability testing of the Scoliometer. Phys Ther, 1990, 70: 108-117. [Medline]

10) Ruggerone M, Austin JH: Moiré topography in scoliosis. Correlations with vertebral lateral curvature as determined by radiography. Phys Ther, 1986, 66: 1072-1077. [Medline]

11) Pearsall DJ, Reid JG, Hedden DM: Comparison of three noninvasive methods for measuring scoliosis. Phys Ther, 1992, 72: 648-657. [Medline]

12) Negrini S, Aulisa AG, Aulisa L, et al.: 2011 SOSORT guidelines: Orthopaedic and rehabilitation treatment of idiopathic scoliosis during growth. Scoliosis, 2012, 7: 3. [Medline] [CrossRef]

13) Scoliosis Research Society: SRS Terminology Committee and Working Group on Spinal Classification Revised Glossary of Terms [Scoliosis Research Society Web site]. March 2000. http://www.srs.org/professionals/ glossary/SRS revised_glossary_of_terms.htm (Accessed Oct. 20, 2012)

14) Lenke LG, Bridwell KH, Baldus C, et al.: Preventing decompensation in King type II curves treated with Cotrel-Dubousset instrumentation. Strict guidelines for selective thoracic fusion. Spine, 1992, 17: S274-S281. [Medline] [CrossRef]

15) Ward WT, Rihn JA, Solic J, et al.: A comparison of the lenke and king classification systems in the surgical treatment of idiopathic thoracic scoliosis. Spine, 2008, 33: 52-60. [Medline] [CrossRef]

16) Hosman AJ, Langeloo DD, de Kleuver M, et al.: Analysis of the sagittal plane after surgical management for Scheuermann's disease: a view on overcorrection and the use of an anterior release. Spine, 2002, 27: 167-175. [Medline] [CrossRef]

17) Deviren V, Berven S, Kleinstueck F, et al.: Predictors of flexibility and pain patterns in thoracolumbar and lumbar idiopathic scoliosis. Spine, 2002, 27: 2346-2349. [Medline] [CrossRef]

18) Schwab FJ, Smith VA, Biserni M, et al.: Adult scoliosis: a quantitative radiographic and clinical analysis. Spine, 2002, 27: 387-392. [Medline] [CrossRef]

19) D'Andrea LP, Betz RR, Lenke LG, et al.: Do radiographic parameters correlate with clinical outcomes in adolescent idiopathic scoliosis? Spine, 2000, 25: 1795-1802. [Medline] [CrossRef] 